Questions vives

\section{Questions Vives}

Recherches en éducation

$\mathbf{N}^{\circ} 34 \mid 2020$

Expériences vécues et surgissement d'événements : une écriture du sensible en recherche biographique

\title{
Prise en compte des expériences vécues : de la chair des corps à l'intercorporéité dans le monde commun de la vie
}

Taking into account lived experiences: from the flesh of bodies to

intercorporeality in the common world of life

\section{Martine Janner Raimondi}

\section{(2) OpenEdition}

Journals

\section{Édition électronique}

URL : https://journals.openedition.org/questionsvives/5213

DOI : 10.4000/questionsvives. 5213

ISSN : 1775-433X

Éditeur

Université Aix-Marseille (AMU)

\section{Édition imprimée}

Date de publication : 18 décembre 2020

ISBN : 978-2-912643-58-2

ISSN : 1635-4079

\section{Référence électronique}

Martine Janner Raimondi, « Prise en compte des expériences vécues : de la chair des corps à

l'intercorporéité dans le monde commun de la vie », Questions Vives [En ligne], № 34 | 2020, mis en ligne le 18 décembre 2020, consulté le 11 octobre 2021. URL : http://journals.openedition.org/ questionsvives/5213; DOI : https://doi.org/10.4000/questionsvives.5213

Ce document a été généré automatiquement le 11 octobre 2021.

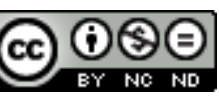

Questions Vives est mis à disposition selon les termes de la licence Creative Commons Attribution -

Pas d'Utilisation Commerciale - Pas de Modification 4.0 International. 


\title{
Prise en compte des expériences vécues : de la chair des corps à l'intercorporéité dans le monde commun de la vie
}

\author{
Taking into account lived experiences: from the flesh of bodies to \\ intercorporeality in the common world of life
}

Martine Janner Raimondi

1 L'enjeu de cet écrit consiste à considérer le rôle et la place du corps dans les expériences vécues narrées dans le cadre de la recherche biographique tant du côté du sujet premier concerné par une enquête, que du côté du chercheur avec lequel il est en dialogue. Ainsi, au-delà de ce que nous pourrions appeler un corps à corps touchant et touché par le récit d'expérience(s) vécue(s) au tréfonds de sa chair, ce seront aussi les lecteurs, chercheurs ou non, qu'il convient de prendre en compte au regard des enjeux mêmes de la diffusion d'une recherche. Apparaissent alors des phénomènes de résonnances depuis la transmission des expériences via des récits oraux, retranscrits ou des écrits jusqu'aux interprétations/traductions faites au cours de leur réception. Deux approches fondamentales sont requises: la phénoménologie et l'herméneutique, notamment en considération des interprétations et reconfigurations du récit lu ou entendu.

\section{Rapport au monde commun de la vie : le Lebenswelt}

2 Considérer en premier lieu l'expérience faite conduit à appréhender la dimension du sensible, comprise comme ce qui se donne à travers le rapport du sujet au monde qui l'entoure, et, au-delà, à d'autres rapports au monde établis par d'autres sujets en d'autres temps, d'autres lieux, y compris ouvrant sur des mondes intérieurs, imaginaires. Pour penser une telle considération, la notion de Lebenswelt de Husserl 
(1935/2012) déclinée en monde commun de la vie partagé sans se réduire, ni au monde objectif de la science, ni à celui contingent des faits, est incontournable. Comme le souligne Depraz (2006), la phénoménologie centrée sur l'engendrement du sens par la conscience fait écho au monde de la vie, appréhendé comme monde commun intersubjectif dans lequel chacun peut avoir part à la vie des autres (Ibid, p. 45).

La dimension du devenir corrélative à la quête de sens qui met la conscience au travail, renvoie à un en-deçà et un au-delà : l'en-deçà, à une infra-conscience; l'au-delà, au monde des autres sujets réunis dans un nous anonyme, qui nous rassemble et nous dépasse, comme aussi bien au monde du vivant. La dimension génétique comporte également une temporalité, qui n'équivaut pas au progrès, mais davantage à l'idée héraclitéenne de flux inexorable du cours de la vie. Si la recherche biographique accorde une place aussi importante à l'expérience du sujet dans le monde sensible de la vie, que nous avons en partage, alors les écrits philosophiques de Husserl et MerleauPonty et leurs commentateurs, attachés à appréhender les expériences comme phénomènes, devraient contribuer à asseoir la posture épistémologique de ce domaine de recherche.

\subsection{Sens de la communauté et corps-de-chair chez E. Husserl}

Pour Rogozinski (2002), Husserl, dès 1907, appréhende les kinesthèses, c'est-à-dire les sensations de se mouvoir en lien avec la constitution perceptive ayant partie liée avec le moi-chair, appelée Ichleib. Puis, en 1913, Husserl met l'accent sur l'importance de la communauté, en développant l'idée que la prise en compte du corps propre suppose le monde de la vie et des vivants. C'est donc la communauté qui fonde le monde de la vie constitué par un nous anonyme. Plus tard, en 1921, dans ses conférences d'où seront issues les Méditations cartésiennes (1929), Husserl reprend la distinction entre le corps de chair, avec le mot Leib, et le corps objectif de la science, qui renvoie au mot Körper (1929, V Véditation).

5 Ainsi le Lebenswelt de Husserl, ce monde de la vie, est, comme le propose de Lavigne (2019), qualifié :

par référence exclusive à l'expérience de la perception du monde ambiant, comme situations perceptive, et par référence à l'expérience subjective qui fonde une telle perception, à savoir le contrôle egoïque spontané des kinesthèses, dans leur articulation fonctionnelle avec [...] le flux des data sensoriels. (Ibid., p. 25).

6 L'expérience subjective du corps-de-chair comporte une dimension kinesthésique reliée et coordonnée à un «Je peux » ouvrant à des potentialités de mouvements. Elle comporte également une dimension d'affectation au sens de réceptivité sensorielle. La phénoménologie donne ainsi une assise au monde sensible compris comme l'ensemble de ce qui apparaitt et qui nous est commun donnant accès aussi bien à l'agent subjectif de la sensation qu'au pouvoir de décision et de contrôle de cet agent et, plus largement aux échanges intersubjectifs selon ces deux modalités. Le sensible n'est donc ni un autre monde, ni un arrière monde, ni même encore un pré-monde; le sensible est le monde commun à tous les vivants.

7 Cet ancrage théorique donne sa légitimité aux approches en recherche qui viseraient à le prendre en compte en donnant à voir et à entendre ses aspects spécifiques et constitutifs. Or, la plupart des travaux scientifiques objectivent les données recueillies, ce qui revient à les considérer uniquement sous la forme d'objets de sciences, que le 
chercheur produit, traite, analyse et commente. La recherche biographique, initiée par Delory-Momberger $(2009 ; 2014)$, a pour objet les processus de construction du sujet au sein de l'espace social à travers le repérage de la compréhension que les individus ont de l'histoire de leur vie et du sens qu'ils donnent à leurs expériences faites dans des environnements historiques, culturels, sociaux, politiques, économiques qui produisent des cadres sociaux. Dès lors, les rencontres et dialogues entre sujets et chercheurs, initiés dans le cadre des recherches de ce type, qui s'appuient fondamentalement sur le partage des expériences de la vie, contribuent d'une part, à ouvrir l'accès à différents mondes; d'autre part, à former le commun du monde de la vie.

\subsection{Corps sujet et corps objet : deux versants d'une même corporéité}

8 Appréhender les différents vécus de conscience permet d'accéder à la vie subjective tout en prenant momentanément une forme objective. Cela vaut pour moi, autant que pour l'autre, perçu comme un corps objectivé perceptible en extériorité avec lequel j'entre en résonnance, tout en restant malgré tout un corps sujet mouvant. L'appréhension de soi comme corps-sujet aussi bien qu'en tant que corps-sujet se retrouve également dans l'appréhension de l'autre, comme autre soi-même. Ces appréhensions occupent le statut d'un événement objectif dans le monde sans pour autant perdre toute trace de subjectivité. En d'autres termes, la vie subjective se retrouve dans le partage intersubjectif des visées objectivantes de soi.

La somatologie développée par Husserl dans Idées III, entendue comme "science du corps vivant » renvoyant à la chair - Leib - n'évacue ni le champ premier, originaire, de la perception via son corps propre, ni le travail de construction de sens accompli par la suite. Dès lors, vivre d'un point de vue phénoménologique revient à éprouver des perceptions somatiques multiples auxquelles sont adjointes des interprétations.

10 La sensibilité s'appréhende à travers le senti, où le soi s'éprouve et se situe dans cette expérience vivante. Pour autant, cette expérience sensible, vécue et première peut être saisie afin d'en tirer ensuite une connaissance objective. Il n'y a pas chez Husserl de séparation radicale, infranchissable entre les deux dimensions. Comme le précise encore Lavigne (2019) :

(...) Mais cette vie organique objective à la fois vivante et vécue, peut devenir en outre objet d'une connaissance objectivante, objet de la 'somatologie', du fait que la saisie interprétative empathique transfère intentionnellement sur le corps-objet d'autrui cette caractéristique éminente d'être auto-donnée à soi-même, de s'éprouver consciemment comme chair. (Ibid, p. 26).

11 En psychiatrie, Brokatski, Gennart \& Thonney (2015) adoptent ce point de vue en développant une attitude dans la façon, pour le soignant, d'être présent en consultation en développant une sensibilité clinique. Cette attitude se traduit par l'attention portée au fondement de l'expérience vécue du patient à travers sa manière d'être au monde, son rapport aux autres, la relation à soi et à l'historicité de sa vie. L'intersubjectivité est ici fondamentale d'autant que la souffrance psychique renvoie à une forme particulière d'isolement. Cette dimension fait écho à la dimension de monde commun de la vie Lebenswelt - en se préoccupant d'aménager un espace de rencontre, qui favorise le dialogue afin que le sens soit partagé. L'enjeu consiste alors à passer d'une situation vécue comme difficile pour la personne qui souffre en lui proposant une situation où elle puisse se sentir reconnue comme ayant sa place dans la « communauté humaine». 
Pour les mêmes raisons, Gennart \& Vannotti (2016) refusent que la thérapie se réduise aux approches objectivantes, qui ne permettent pas d'appréhender le trouble douloureux chronique dans sa réalité phénoménologique » (Ibid., p. 147).

Pour autant, appréhender les profondeurs du vécu n'est pas sans difficulté, notamment lorsque les expériences éprouvées confinent à l'étranger, voire à l'indicible.

\subsection{Expérience de l'étrangeté à soi, en soi}

13 La vie rattachée à un corps perceptible de l'extérieur peut traverser des épreuves et faire des expériences qui viennent perturber cette vie organique subjective, vivante et vécue, au point de mettre en déroute le sujet. Rogozinski (2019) commente l'idée d'effondrement total de la communauté intersubjective formulée par Husserl, en 1931 :

(...) Normalement, l'existence humaine demeure dans cette normalité, malgré des entraves occasionnelles, des perturbations, des ruptures (...) à nouveau dépassées. Mais n'est-il pas aussi possible cependant que le cours du destin soit tel qu'il briserait totalement cette normalité et me placerait dans une situation où je devrais dire : « je ne sais plus où j'en suis, je ne parviens pas à voir comment la vie pourrait continuer » ? Est-ce qu'un effondrement total de la communauté humaine n'est pas possible ? : (Rogozinski, Ibid., p. 213).

Ainsi, l'irruption d'un événement « anormal » peut provoquer une crise du monde de la vie au point de l'anéantir, bien au-delà des dissonances entre les esquisses perceptives toujours possibles entre elles. Pour Rogzinski, l'expérience de «situations limites de l'existence ", dont on ne revient pas indemne, conduit les personnes, qui les vivent, à ne plus savoir quel sens donner à ce qu'elles vivent, pouvant aller jusqu'à ne plus vouloir vivre. De tels événements peuvent provoquer différentes formes d'écart: déviation, bifurcation, voire, rupture radicale dans le cours de la vie pouvant aller jusqu'à la mort. La possibilité de trouver son chemin de Damas, compris comme intime conviction d'avoir trouvé sa voie, peut néanmoins apparaître, avec la conviction intime d'être dans le juste, plutôt que suite à un raisonnement rétroactif.

Dans le cas d'une situation limite, la perte de sens et de repères est telle, qu'il s'agit plutôt d'un vécu en ligne de crête, aux bords du précipice, risquant à tout moment de faire basculer le soi, avec l'impression irrévocable d'un effondrement du monde commun de la vie autour de soi. Le monde de la vie - Lebenswelt - ne fait plus sens et le sujet ne se reconnaît plus comme appartenant à ce monde. La part du commun et l'enjeu de communauté qu'il comporte se délitent au point de faire perdre au sujet sa substance vitale elle-même.

Les catastrophes naturelles peuvent provoquer de tels effets, mais les violences humaines, de la plus insidieuse à la plus cruelle peuvent également être traumatiques. De tels moments entraînent une sidération, au cours de laquelle la personne qui s'y trouve confrontée, se sent annihilée, comme si le sol s'effaçait sous ses pieds. Lorsque la maladie grave ou le handicap survient, nombre de personnes expriment ce sentiment d'écroulement, qu'elles nomment "catastrophe », "tsunami », avant de tâcher de se relever, ou non.

17 Si l'on admet avec Husserl que les faits sont contingents, ce serait précisément cette contingence qui accentuerait la souffrance des personnes. La recherche E-Plateform Innov (2016-2018) ${ }^{1}$, à laquelle nous avons participé, visait à appréhender les expériences vécues par des personnes atteintes de maladies rares, systémiques, auto- 
immunes comme le Lupus ou la sclérodermie et à repérer les ressources mobilisées mais également les freins, voire les situations de handicap rencontrées, en lien avec le vivre avec l'une de ces deux maladies. La méthodologie adoptée a consisté à solliciter tout d'abord le récit écrit de : «Comment tout cela a commencé ?», déposé sur une plate-forme numérique collaborative, censée, grâce à ce partage, contribuer à créer du commun, notamment face à l'isolement, voire à l'invisibilité ressentie par les patient.e.s.

Concernant l'événement de la survenue de la maladie, les personnes ont d'emblée posé la question : «pourquoi moi ?». Car, loin de se satisfaire de l'idée d'un destin, a fortiori malveillant, il s'agissait de comprendre quel sens pouvait avoir cet événement vécu sur le mode d'une incompréhension radicale. La souffrance liée à cet événement était d'autant plus grande que la maladie elle-même n'était le plus souvent pas connue des médecins généralistes eux-mêmes. Les personnes ont exprimé ce que Benjamin (1933/2018) avait repéré à propos de la "pauvreté d'expérience » - autrement dit, du manque de partage d'expériences vécues - pouvant ainsi altérer l'humanité au point de développer une nouvelle barbarie (Janner Raimondi, 2020). Précisément, le partage d'expériences vécues entre les sujets humains contribue à développer une communauté de savoirs qui permet aux malades de continuer à se sentir appartenir à cette communauté. L'importance de ce partage d'expériences des maladies va de pair avec le partage de la connaissance médicale de ces maladies. En effet, l'errance thérapeutique de quelques semaines à plus de deux ans - exprimée par plusieurs patients, fut une expérience particulièrement éprouvante, soulignant ainsi l'importance de l'acte de nommer les choses, moins pour s'arrêter au nom lui-même, que pour raccrocher ce que le sujet vit dans sa chair à un phénomène identifié et connu par d'autres. Comme l'a précisé Camus (2006) : "Mal nommer un objet, c'est ajouter au malheur du monde » (2006, vol. 1, p. 908). Cette quête de la vérité, même si elle s'avère tragique, importe d'autant que la conviction que "parler répare» (Camus, 1947, p. 25). Ne pas pouvoir mettre de mots sur un vécu de maladie accentue l'angoisse de la mort qui rôde. De facto, lorsque les patients ont vu leur douleur et leur souffrance étiquetées par un nom de maladie, cela n'a pas manqué d'avoir, tout au moins au début, un effet «libérateur » dans la mesure où ils n'étaient plus des malades imaginaires. Poser le diagnostic d'une maladie, fût-elle rare, permet aux sujets qui vivent avec, de se sentir moins isolés et en partie reconnus. L'importance du sentiment d'appartenance à une communauté élargie est apparue fondamentale pour (re)mobiliser leur énergie vitale et combattre la maladie, selon une métaphore militaire (Sontag, 1977/2009). Dans cette recherche, l'écoute des personnes malades a permis d'identifier combien le corps-sujet de chair a besoin du corps-objet de connaissances, renforçant par là-même l'idée même d'une vie subjective et intersubjective.

\section{L'événement d'une maladie qui survient et vécu de l'essence de cette maladie dans un corps de chair}

19 Dans le sillage de Husserl, nous considérons que la contingence des faits n'empêche pas l'essence de ces derniers. Pour les malades atteints du Lupus ou de Sclérodermie, le fait de tomber un jour malade corrobore l'idée d'une contingence radicale. En effet, ils auraient pu ne pas contracter la maladie et/ou ne pas la développer, dans la mesure même où il n'y avait pas de nécessité causale d'être malade. 


\subsection{De la contingence des faits vécus à l'appréhension de leur essence et construction du sens de l'événement}

Si le fait de tomber malade relève d'une contingence, la question de l'essence même de la maladie renvoie : d'une part, à la nosographie médicale; d'autre part, aux formes concrètes prises par la maladie au niveau du corps de chair du sujet. En lien avec Husserl, nous comprenons ainsi la signification entre contingence de fait et essence : alors que la contingence renvoie à l'absence de nécessité qu'une chose existe ou non n'invalide pas l'idée que tout phénomène existant comporte une essence, un eidos comportant différents degrés de généralité.

Un objet individuel n'est pas simplement un individu en général, un « ceci, là ! », un cas unique ; en tant qu'il possède " en lui-même » tels ou tels caractères internes, il a son type propre (Eigenart), sa réserve permanente de prédicables essentiels, qui doivent lui échoir (comme étant tel qu'en lui-même il est), pour que d'autres déterminations, secondaires, relatives, puissent lui échoir : (Husserl, 2018, p. 24).

Les expériences vécues fonctionnent comme des analyseurs au sens de Lapassade (1971); potentiellement, elles révèlent l'essence de l'expérience considérée. Si l'événement renvoie à une temporalisation des phénomènes, toute expérience vécue comme événement n'engendre pas toujours des réorientations du cours de la vie. Parfois, même, ce n'est que bien plus tard que le sujet développe une prise de conscience des étapes, sinuosités ou ruptures dans son parcours. Considérant la question de l'expérience comme événement qui advient et duquel potentiellement s'opère un déploiement qui diffère du parcours suivi jusque-là, différents types d'événement apparaissent :

- l'événement "pierre angulaire» d'un parcours de la vie, qui fournit au sujet humain l'occasion de donner à voir la force de son être intérieur, et qui ne manque pas de surgir, parfois même contre toute attente ;

- l'événement "pierre de touche ", qui permet de révéler les composants de sa vie, à l'instar du procédé utilisé pour vérifier le titrage d'un alliage ou d'un métal précieux en orfèvrerie ;

- l'événement "pierre d'achoppement », sur lequel le sujet humain butte, au risque de se perdre.

De facto, il est des situations qui pour certains sujets occuperont davantage le rôle de "pierre angulaire», que de "pierre de touche» ou de pierre d'achoppement». La situation vécue, selon son retentissement sur le soi profond corrélé à l'environnement du moment pourra être dite contingente et pourtant renvoyer à l'essence propre du sujet humain qui la vit sans pour autant réduire ce qu'il vit comme s'il était le seul à le vivre. C'est dans ces moments-là que le Lebenswelt, le monde commun de la vie se trouve brisé pouvant conduire le sujet humain qui vit soit au mutisme, soit à l'isolement avec l'expression " tu/vous ne peux/pouvez pas comprendre ", soit encore à la colère. Pour Rogozinski (2019) :

(...) la communauté intersubjective est originairement constituante, mais notre mode d'accès à cette communauté passe nécessairement, pour chacun d'entre nous par un ego singulier, à chaque fois le mien, et par la constitution « en moi et par moi » de l'alter ego : (Ibid., p. 93). 


\subsection{La parole est à Chahida}

23 Le récit restitué ci-dessous, Chahida ${ }^{2}$, atteinte du Lupus, donne chair aux propos formulés ci-dessus. Il révèle ses douleurs et ses efforts inouïs pour tâcher de préserver ce qu'elle appréhende comme le monde commun de la vie quand on est jeune et que l'on a la vie devant soi. Ce monde commun dans lequel elle a grandi, jusqu'alors, n'est pas sans résonnance avec le mode habituel de vie tissé de rapport aux autres. 
Un matin, en 1984 j'avais alors 19 ans, je voulais transvaser du lait dans une casserole pour le faire bouillir, malheureusement j'ai tout lâché car je n'avais plus de force dans le poignet, et en le touchant c'était très chaud, et pourtant aucune douleur ne m'avait alertée au préalable. J'ai donc consulté un rhumatologue en Algérie (j'y vivais à l'époque) qui a longtemps hésité entre une PR et un RAA, sans pour autant lésiner sur les corticoïdes et les infiltrations, mes douleurs devenaient atroces et générales, non seulement je ne pouvais pas marcher, mais j'ai commencé à avoir des lésions cutanées au niveau des doigts et ce fameux masque rougeâtre en aile de papillons sur le visage. Voyant ma souffrance, et le désarroi de ma famille face à cette impuissance doublée de l'incapacité et de l'ignorance des médecins, ma tante a été informée qu'il était possible de venir à Paris et consulter l'illustre Professeur Amor, à l'époque, chef de service de rhumatologie à Cochin; et après une longue hospitalisation et maints examens, plus une biopsie le verdict est tombé: Mademoiselle vous avez un LUPUS.

Ma lutte ou plutôt, mon duel avec le LOUP (lupus) a commencé.

Que vous dire? Quand on a à peine 19 ans et que l'on pense que l'on a toute la vie devant soi, je n'ai pas réalisé ni mesuré l'ampleur de cette maladie, jusqu'alors méconnue et surtout en Algérie!!! pendant mon hospitalisation à Cochin le Pr Amor et son équipe m'ont bien expliqué les différentes phases du traitement et jeétais rassurée , du moment que je n'avais plus mal, et que j'étais entre de bonnes mains, je voulais guérir et terminer mes études de chirurgie dentaire, je pensais que le pire était déjà passé , ma famille ne comprenait pas tout mais me soutenait et m'encourageait à rester aussi longtemps que nécessaire pourvu que je guérisse. Je gonflais à vue d'œil avec la corticothérapie et j'étais essoufflée mais c'était pour la bonne cause, je crois que je n'ai jamais ressenti ça comme une tare ni une fatalité, ma foi en Dieu était et est toujours sans équivoque, Dieu donne la maladie et peut tout aussi bien la reprendre, il suffisait d'y croire et de s'y accrocher.

Hélas , j'ai déchantée très vite en rentrant en Algérie, mon fiancé de l'époque n'a rien compris et n'a pas accepté mon nouveau corps, les étudiants me regardaient tantôt avec pitié tantôt avec méfiance, et pour tout le monde j'étais condamnée par un cancer incurable, sinon que j'étais contagieuse....j'ai subi des méchancetés gratuites et des injustices par des êtres ignares et bornés, c'est alors que j'ai décidé de faire le ménage dans ma vie et que la bêtise humaine ne m'atteindrait plus jamais, je me suis fixé pour objectif de réussir mes études haut la main malgré les contraintes de mes déplacements fréquents à Paris, je révisait à l'hôpital et je passais les examens ratés en rattrapage, je me suis créé mon propre univers avec ma famille proche et je subissais tous les effets du Lupus avec résignation, nous avons enfin appris à coexister. Et je devins enfin Docteur en chirurgie dentaire avec félicitations du jury ; Ma première victoire contre le LOUP (...).

En voyant mon combat contre les douleurs, mon silence et ma résignation, ma famille ne se donnait plus le droit de se plaindre, et je me plais à espérer que je leurs ai insufflé le courage, et surtout de relativiser certains évènements qui semblaient à leurs yeux dramatiques.

(..) Je me suis dit, il faut apprendre à connaitre cette maladie pour pouvoir l'apprivoiser, et apprendre à y faire face en toutes circonstances, (ne doit-on pas connaitre ses ennemis pour pouvoir mieux les combattre); et je ne me suis pas privée de poser toutes les questions qui me tracassaient, au Pr Amor, il était mon unique interlocuteur et je lui faisait confiance, c'est très important de se fier complètement à son médecin car si on y croit ça marche mieux, je n'ai jamais été chercher des réponses sur internet on y trouve tout et n'importe quoi, et souvent les informations sont erronées, et c'est l'angoisse totale, c'est pour cela que j'ai toujours privilégié le contact humain.

Il est important de savoir à quoi s'attendre, si j'étais resté dans l'ignorance, je crois que je ne serai plus de ce monde, à chaque crise et chaque nouveau symptôme, je demandais au professeur s'il avait déjà vu ça, si oui cela me rassurait, mais si non et cela a souvent été le cas pour moi, je lui demandait qu'elle solution il préconisait, car je savais ou du moins je me persuadait qu'il y avait toujours une solution; parfois même j'acceptais de servir de cobaye, et je servais de patient modèle dans l'amphithéâtre du CHU, pour faire avancer les recherches et permettre à tout le monde de trouver.....(plus on est de fous....). Hormis tout cela, je voulais me battre pour mes enfants, ils n'avaient plus que moi, et il était hors de question que je baisse les bras, j'avais trop souffert pour les avoir, et si le Bon Dieu l'a permis ce n'était surement pas pour qu'ils grandissent orphelins de père et de mère! (...) Vous imaginez donc aisément ma hargne à gagner cette bataille contre LE LOUP et ma rage de vivre, car quand on a autant souffert, on n'a pas le droit de baisser les bras; je ne m'octroie pas le droit de laisser tomber et croyez-moi, devant la perte des êtres que l'on aime, mes crises et mes douleurs sont une partie de plaisir.

gleestionsdtisést, l2020us disant que jamais avant 2015, je n'ai eu l'opportunité de parler de ma maladie, ce n'est qu'après m'être inscrite à l'association 'Lupus Plus' que j'ai rencontré pour la première fois des patients avec la même maladie et j'avoue que cela m'a soulagée de savoir que je n'étais pas la seule, car ce sentiment d'isolement était présent, et j'ai vu qu'ils ne réagissaient pas toujours bien, face à leur maladie, il y en avait qui étaient perdus, et j'avoue que ce jour-là j'ai été heureuse de voir que j'avais surmonté ces angoisses et 

vécu de chair souvent diffus appréhender et appréhender le corps propre comme unité ? Cela supposerait que soit suffisamment constitué un schème du corps propre ou bien encore que ce schème ne soit pas altéré. Il y aurait ainsi comme un mouvement dialectique entre d'une part, les vécus de chair visés par la conscience dans un cadre temporel qui invite à considérer le présent vivant sous la forme du tout juste passé et le tout juste à venir (Farges, 2019) et, d'autre part, un rapport de transférence ${ }^{3}$ (Rogozinski, 2019) entre l'expérience perçue du vécu et le corps. Le corps comme enveloppe unitaire rassemble les perceptions du vécu de chair et rend ainsi possible non seulement la prise de conscience mais également la réflexion comprise comme retour sur soi de l'expérience. Le corps, à ce titre, rend possible la localisation de la perception en signifiant que le local interfère avec le global et réciproquement. En effet pour Rogozinksi, si le corps permet de situer la perception en son sein, celle-ci contribue à renforcer, à affiner l'appréhension du schème. Il s'agit moins, dans ce cadre, d'une appréhension globale du corps que d'une opération de spécification. Pour 
le dire autrement, les perceptions locales de la chair contribuent au développement d'une appréhension de son propre corps, c'est-à-dire du corps de chair réuni, unifié, englobé d'un soi unique. Dans le cas de la maladie chronique, il est des situations extrêmes, qui telles des événements de type pierre d'achoppement rendent particulièrement difficiles le rapport au corps propre dans son unité constitutive et par là-même, rendent quasi impossible le rapport à la conscience de soi et le rapport réflexif à soi, comme dans le cas des sidérations traumatiques.

\section{Expérience de la résistance des phénomènes : approcher l'invisible}

Loin d'en rester à l'attitude naturelle qui d'emblée projette sur les choses, les opinions du sens commun ou les interprétations habituelles, la "somatologie» suppose une démarche que Husserl nomme la Réduction phénoménologique rendue possible grâce à la mise en œuvre de l'épochè comprise comme suspension et mise entre parenthèses des convictions et des croyances de l'attitude naturelle pour parvenir à la description des vécus de conscience. Les expériences vécues de chair demandent à être reliées à mon corps pour favoriser ma propre incorporation qui rend à son tour possible la transférence de Rogozinski, dans une relation de type analogique entre soi et l'autre. Car ma chair reste incomplète si elle ne fait pas corps avec soi, avec l'autre. En retour, si rien ne ressemble à ma chair et par extension, à mon corps, alors la dissemblance du corps de l'autre peut conduire à une désorientation fondamentale, une perte du monde commun de la vie. Pour Rogozinski (2006), la dissemblance du corps de l'autre porte en elle la racine du dégoût ou de la haine précisément parce que le corps de l'autre ne peut être mis en communauté avec soi. Par là-même, il est inassimilable. Or, cette mise en commun du corps d'autrui constitue un phénomène instable et précaire, se rejouant à chaque instant. Il en est de même pour mon propre corps, qui, lorsqu'il se désincorpore, peut lui aussi se vivre et être vécu aux limites de la communauté humaine.

Merleau-Ponty (1945) a développé la pensée de Husserl notamment en ce qui concerne la perception originaire, pré-objective et pré-consciente, qui relève d'un sentir incarné : "je sens qu'on me sent et qu'on me sent en train de sentir et en train de sentir ce fait même qu'on me sent » (1945, p. 187). Berthoz (2010) saluait sa conception multimodale de la perception. Retenons que le mouvement de conscientisation ne saurait s'appréhender sans le sujet sensible mêlant perceptions, intuitions et réflexion sur fond d'être de chair, corrélé à l'élaboration du schème de son corps propre en écho aux dimensions bio-psycho-sociale de la vie en commun.

Dans ses notes de 1959, Merleau-Ponty reprend la démarche husserlienne d'une considération des phénomènes et invite son lecteur à considérer que "voir le monde " suppose en réalité " d'apprendre à le voir », c'est-à-dire prendre conscience de ce nous y projetons avant même de nous en rendre compte, mais également se saisir de ce que suppose l'acte de voir le monde.

(...) Déjà mon corps, comme metteur en scène de ma perception, a fait éclater l'illusion d'une coïncidence de ma perception avec les choses mêmes. Entre elles et moi, il y a désormais des pouvoirs cachés, de fantasmes possibles qu'il est qu'il ne tient en respect que dans l'acte fragile du regard : (1959/1964, p. 23-24). 
Lerleau-Ponty permet de dépasser la seule prise en compte de l'intériorité, propre à la subjectivité, car le sujet humain est ipso facto dans le monde ; il y est pris et y participe. Dans son dernier ouvrage, Merleau-Ponty écrit : «Or, cette certitude injustifiable d'un monde sensible qui nous soit commun, elle est en nous l'assise de la vérité » (Ibid., p. 27). Ainsi, le monde sensible, loin de se réduire à n'être qu'un objet, comprend fondamentalement le vivant et sa dynamique; il est le socle commun dans lequel via les perceptions puis la réflexion, nous forgeons nos idées de vérité.

corps est considéré alors comme ce qui permet d'explorer le monde et renvoie au concept husserlien de corps de chair. Mais, par de-là, le corps de chair et le monde de la vie que les êtres vivants ont en commun, Merleau-Ponty développe le phénomène d'enracinement sur la Terre qui nous nourrit, nous façonne, sur laquelle les expériences elles-mêmes se forment. Nos rapports à la Terre, au monde et aux autres ne sont ni déterminés, ni fermés et les questions que les êtres humains se posent font écho à leurs quêtes de sens. Il précise qu'il ne saurait y avoir de non-sens aux questions que l'homme se pose, ni même d'ailleurs de solutions préalables mais bien plutôt un horizon de sens. En effet, le langage n'épuise pas les choses; il ne fait que les approcher sans cesse. Ainsi, la vérité de l'existence, toujours approximative entre hésitation et prudence, diffère fondamentalement d'une équation mathématique. Pour MerleauPonty, la vérité c'est l'invisible, qui fait apparaître derrière le phénomène quelque chose qui, en retour, fonde le phénomène et ne sera jamais visible. Il y a comme une résistance du phénomène, qui ne peut faire qu'il devienne totalement objet. Quelque chose toujours échappe, comme une persévérance de l'être du phénomène alors même que les œuvres produites à son propos en disent quelque chose. L'évolution de la pensée de Merleau-Ponty nous invite à considérer davantage l'invisible, que le visible. Pourquoi?

Réfléchir n'est pas coïncider avec le flux depuis sa source jusqu'à ses ramifications dernières, c'est dégager des choses, des perceptions, du monde et de la perception du monde, en les soumettant à une variation systématique, des noyaux intelligibles qui lui résistent, cheminer de l'un à l'autre d'une manière que l'expérience ne dément pas, mais qui ne nous donne que ses contours universels, qui donc laisse intact par principe le double problème de la genèse du monde existant et de la genèse de l'idéalisation réflexive, et enfin évoque et exige comme son fondement une surréflexion où les problèmes derniers seraient pris au sérieux : (Ibid., p. 69-70).

La problématique soulevée par la pensée du visible et de l'invisible permet de comprendre qu'il existe toujours un dépassement de la conscience de soi comme maître des choses. Car ce que je suis au total déborde ce que je suis pour moi. Ce qui pouvait apparaitre comme l'empire de la conscience, doit être revu, car l'au-delà des phénomènes du monde vivant présente une résistance à tout enfermement. Inaugurant une phénoménologie de l'autre monde Merleau-Ponty permet de considérer combien l'expérience du visible ou du toucher comme l'expérience du monde sensible en général ne se résume jamais à la chose que l'on voit, que l'on touche; il y a un fond, un socle, un quelque chose que l'on ne voit pas.

Ce n'est pas quelque chose qu'on verra après, c'est ce qui rend visible, toujours mobile. Le visible est doublé par quelque chose qui en fait son essence, le visible est inépuisable, 
il ne se réduit pas à une forme, il est comme alimenté par une source: l'être. Le sensible, le visible doit être l'occasion de dire l'invisible. L'invisible renvoie ainsi à :

1. ce qui n'est pas actuellement visible, mais pourrait l'être (aspects cachés ou inactuels de la chose, - choses cachées, situées « ailleurs » - « Ici » et « ailleurs »;

2. ce qui, relatif au visible, ne saurait néanmoins être vu comme chose (les existentiaux du visible, ses dimensions, sa membrure non figurative);

3. ce qui n'existe que tactilement ou kinesthésiquement etc.;

4. les $\lambda \dot{\varepsilon} x \nmid \alpha$, le Cogito : (Ibid, p. 305).

Lorsque l'on écrit, c'est l'expérience du sujet à travers son corps propre qui transparaît dans l'écriture, comme si la pensée devenait alors corps, matière. Au lieu de penser qu'avec la conscience, je domine le monde, il s'agit d'appréhender combien je suis du monde, combien j'en fais partie. Notre rapport au monde est un rapport de perception via un corps peu à peu individué, explorant de multiples perceptions dans l'expérience sensible mais l'essentiel pour Merleau-Ponty consiste à se ressaisir du lien natal entre le monde et moi, entre moi et autrui. Le travail d'objectivation et de qualification du monde, comme du corps - Körper - ne permet pas d'appréhender l'Être du monde, son ontologie. Mieux, l'être dans sa résistance même aux travaux de connaissances, renvoie pour Merleau-Ponty à l'être sauvage, caché, infini et transcendant.

Ce que je veux faire, c'est restituer le monde comme sens d'Être absolument différent du "représenté", à savoir comme l'Être vertical qu'aucune des «représentations » n'épuise et que toutes "atteignent», l'Être sauvage: (Ibid., p. 301).

Lorsque le sujet fait l'expérience sensible d'autrui ou d'une chose, il s'agit d'une expérience émotionnelle et "presque charnelle où les idées- celles d'autrui et les nôtres - sont moins comprises, qu'accueillies ou repoussées dans l'amour ou la haine » (Ibid., p. 28). Ainsi l'expérience sensible renvoie à une expérience du monde vivant, qui conduit le sujet à sortir de sa seule subjectivité, pour découvrir quelque chose de l'ordre de l'être. De cette rencontre charnelle entre soi-même et l'être - sauvage par nature - naîtrait la chair du monde.

Dès lors, appréhender l'expérience sensible conduit à la dire sans pour autant se satisfaire de cette expression. Comme Merleau-Ponty, Barbaras (1992) développe l'idée d'une non saisie complète de l'expérience sensible via le langage, précisément parce que «ma chair n'est pas ce corps objectif qu'une conscience viendrait animer, mais le point d'origine, le foyer de la phénoménalisation " (Ibid., p. 36). Les événements qui surviennent, s'appréhendent en recherche, lors des rencontres entre la personne concernée par l'enquête et le chercheur, dans le dialogue noué, traversé de relations sensibles via leur inter corporéité.

\section{Conclusion}

Cette approche phénoménologique et herméneutique de l'expérience vécue propose un cadre de référence pour la recherche biographique en éducation-formation, qui invite les chercheurs recourant aux récits biographiques à appréhender les enjeux souvent infra perceptibles d'une considération du corps de chair dans un rapport au monde commun. Par-delà, l'évitement du risque d'oublier la place du sensible en recherche, il s'agit de considérer les fondements théoriques propres à rendre compte de la possibilité même d'une intercompréhension aussi bien entre les chercheurs et les 
personnes concernées par une étude, qu'au niveau de chacune des personnes, y compris au niveau des lecteurs. In fine, cet article, non seulement conceptualise l'intérêt d'une approche phénoménologique du vécu biographique en recherche biographique, mais encore souligne combien l'expérience du monde vécu via le corps propre est inscrite à l'origine et au cœur même de toute connaissance objective. Celle avec laquelle la rationalité scientifique doit composer, sous peine de passer à côté de son objet ouvrant ainsi la voie vers la reconnaissance d'une connaissance hybride entremêlant subjectivité/intersubjectivité et objectivité.

\section{BIBLIOGRAPHIE}

Augustin. Les confessions, Livre Dixième, trad. M. Moreau (1864). Edition numérique réalisée par l'abbaye Saint benoit de Port-Valais.

Barbaras, R. (1992). Motricité et phénoménalité chez le dernier Merleau-Ponty. In : M. Richir \& E. Tassin (Dir.). Merleau-Ponty, phénoménologie et expériences. (p.7-56) ; Paris : Ed. Million.

Benjamin, W. (2018). Expérience et pauvreté [1éd. 1933]. Suivi de Le conteur et La tâche du traducteur. Paris : Payot \& Rivages.

Berthoz, A. (2010). La conscience du corps. In : A. Berthoz \& B. Andrieu (Dir.) Le Corps en Acte. (p. 9-22). Nancy : PUN.

BrokatskY, C., Gennart, M. \& Thonney, J. (2015). L'attitude phénoménologique dans la pratique clinique. Psychothérapies, 1, 22, 21-28.

Camus, A. (2006). CEuvres complètes I, II. Paris : Gallimard.

Camus, A. (1947). L'homme révolté. Paris : Gallimard.

Danou, G. (2008). Peser les mots (Actes du colloque Littératures et Médecine, 2007, université de CergyPontoise), Limoges : Ed. Lambert-Lucas.

Depraz, N. (2006). Comprendre la phénoménologie. Une pratique concrète. Paris : A. Colin.

Farges, J. (2019). Husserl : phénoménologie et fondement des sciences. Paris : Hermann.

Gennart, M. \& Vannotti, M. (2016). Corps et histoire de vie. La Maladie chronique : un défi pour les soignants et pour les proches. Paris : Fabert.

Gennart, M. \& Vannotti, M. (2014). Espaces familiers et identité : quand l'espace propre est hanté. Thérapies familiales, 2014/4, vol. 35, 439-450.

Housset, E. (2020). Cours d'agrégation sur « la représentation » en phénoménologie de Husserl à Levinas. https://hal-normandie-univ.archives-ouvertes.fr / hal - 02881077/document

Husserl, E. (2019). La Phénoménologie et les fondements des sciences. Trad. J. Farges \& D. Pradelle. Paris : Hermann.

Husserl, E. (2018). Idées directrices pour une phénoménologie pure et une philosophie phénoménologique. Trad. J. f ; Lavigne. Paris : Gallimard, $1^{\circ}$ éd. 1913 
Husserl, E. (2012). Conférence : « La crise de l'humanité européenne et la philosophie » Vienne, le 7 mai 1935. Introduction, commentaire et traduction par N. Depraz. Edition numérique : Pierre Hidalgo. La Gaya Scienza, mars 2012. [Consulté le 22 avril 2014 sur : www.ac-grenoble.fr/ PhiloSophie/file/husserl-depraz.fr].

Husserl, E. (2008). Méditations cartésiennes. Trad. E. Levinas \& M. Pfeiffer. Paris : Vrin, $1^{\circ}$ éd. 1929. Janner Raimondi, M. (2020). Récits de personnes atteintes du lupus, maladie auto-immune rare : quels points d'attention ? Corps, 2020, $\mathrm{n}^{\circ} 18,153-162$.

Kittay, E.F. (2015). Le désir de normalité. Quelle qualité de vie pour les personnes porteuses d'un handicap cognitif sévère? Alter, 9/2015, 175-185.

Lapassade, G. et Celma, J. (1971). L'analyseur et l'analyste. Paris : Gauthiers-Villars.

Lavigne, J-F. (2019). Lebenswelt et processus vitaux. In : Y-C. Zarka \& A. Zafrani (Dirs.). La phénoménologie et la Vie. Paris : éditions du Cerf.

Merleau-Ponty, M. (1960). Signes. Paris : Gallimard.

Merleau-Ponty, M. (1964). Le visible et l'invisible. Texte établi par Claude Lefort. Paris : Gallimard, $1^{\circ}$ éd. 1959.

Merleau-Ponty, M. (1945). Phénoménologie de la perception. Paris : Gallimard.

Parain, B. (1943/1972). Recherches sur la nature et les fonctions du langage. Paris : Gallimard, $1^{\circ}$ éd. 1943.

Rapport de recherche (2019) : Récits des Co-chercheurs Recherche E-Plateform-Innov (2016-2018). Dir. Pr. C. Delory-Momberger, Université de Sorbonne Paris Nord, Experice..

Rogozinski, J. (2006). Le Moi et la chair. Introduction à l'égo analyse. Paris : éd. Labyrinthe.

Rogozinski, J. (2019). L'expérience de l'étranger. In : Y-C. Zarka \& A. Zafrani (Dir.). La

phénoménologie et la vie. (pp. 91-123). Paris : éd. du Cerf.

Sontag, S. (2009). La maladie comme métaphore. Paris : Seuil, $1^{\circ}$ éd. 1977.

\section{NOTES}

1. Quatre équipes étaient réunies autour de ce projet : une équipe médicale dirigée par D. FargeBancel; deux équipes en sciences humaines et sociales : l'une, dirigée par C. Tourette-Turgis de l'Université de Paris 6 ; l'autre, dirigée par C. Delory-Momberger de l'Université de Paris 13 ; et une dernière équipe, constituée de la présidente de l'Association Française du Lupus et autres maladies auto-immunes, M. Rivière et du Docteur B. Lantz.

2. Chahida fait partie des 10 patients atteints du Lupus, qui ont accepté d'écrire le récit de leur maladie dans le cadre de la recherche E-Plateform Innov dirigée par Pr. C. Delory-Momberger de l'Université de Sorbonne Paris Nord (2016-2018). Je la remercie encore vivement pour son écrit.

3. Le choix du mot "tranférence" s'explique par le souhait de l'auteur de ne pas risquer d'entretenir une confusion avec celui de "transfert " particulièrement fondamental dans le champ de la psychanalyse. Il s'agit de transférer l'expérience du corps de chair vécue au corps, sans pour autant renvoyer à une explication d'ordre psychanalytique. 


\section{RÉSUMÉS}

Prendre en compte et rendre compte des expériences vécues par le sujet en recherche biographique engage une réflexion d'ordre phénoménologique, inspirée de Husserl et, notamment, de la lecture pragmatique établie par Depraz (2006), visant autant à "voir» l'expérience, qu'à «interroger " les préjugés (Ibid., p.4). Or, accéder aux phénomènes de l'expérience vécue ne saurait être immédiat; leur accessibilité exige une démarche afin de dépasser l'attitude naturelle développée à travers la projection d'idées préconçues ou d'interprétations préétablies. La question se pose autant du côté de la personne directement concernée par une recherche, que du côté du chercheur avec lequel elle est en dialogue, car immanquablement surgissent des éléments perceptifs, sensibles, imaginatifs plus ou moins reliés aux souvenirs en lien avec le corps de chair de chacun et les interactions qui s'y jouent. C'est précisément la considération du corps dans un rapport au monde commun de la vie, qui est étudiée dans cet article afin de rendre compte de l'intérêt d'une approche phénoménologique de l'expérience vécue en recherche biographique ouvrant ainsi la voie à une connaissance hybride où se tissent rationalité scientifique, subjectivité et intersubjectivité.

Taking into account and reporting on the subject's experiences in biographical research involves a reflection of a phenomenological nature, inspired by Husserl and, in particular, the pragmatic reading established by Depraz (2006), aiming as much to "see" the experience as to "question" the prejudices (Ibid., p. 4). However, access to the phenomena of lived experience cannot be immediate; their accessibility requires an approach that goes beyond the natural attitude developed through the projection of preconceived ideas or pre-established interpretations. The question arises as much on the side of the person directly concerned by a research project as on the side of the researcher with whom he or she is in dialogue, because perceptive, sensitive and imaginative elements inevitably arise, more or less related to memories in connection with the body of flesh of each person and the interactions that take place within it. It is precisely the consideration of the body in relation to the common world of life that is studied in this article in order to demonstrate the interest of a phenomenological approach to lived experience in biographical research, thus opening the way to a hybrid knowledge in which scientific rationality, subjectivity and intersubjectivity are woven.

\section{INDEX}

Mots-clés : expérience, sensible, corps, phénoménologie, recherche biographique

Keywords : experience, sensitive, body, phenomenology, biographical research

\section{AUTEUR}

\section{MARTINE JANNER RAIMONDI}

Professeur en Sciences de l'éducation, Université Sorbonne Paris Nord, EXPERICE 